tuée du cautionnement restera définitivement acquise à la commune.

Art. 32. - AGEnts du ConCEssionnaire. - Les agents et gardes que le concessionnaire aura fait assermenter pour la survenllance et la police de la distribution et de ses dépendances seront porteurs d'un signe distinctif et seront munis d'un titre constatant leurs fonctions.

ART. 33. - Cession ou Modification de la ConCEssion. - Toute cession partielle ou totale de la concession, tout changement de concessionnaure ne pourront avoir lieu, à peine de déchéance, qu'en vertu d'une autorisation résultant d'une diélibération du Conseil municipal approuvée par le préfet.

ART. 34. - JUGEMENT DE CONTESTATIONS - Les contestations qui s'élèveraient entre le concessionnaire et l'Administration, au sujet de l'exécution et de l'interprétation des clauses du présent cahier des charges, seront jugées par le Conseil de préfecture du département d...., sauf recours au Conseil d'Etat.

Art. 35. - Election de domicile. - Le concessionnaire devra faire election de domicile à...

Dans le cas où il ne l'aurait pas fait, toute notification ou signification à lui adressée sera valable lorsqu'elle sera fatte au secrltbtariat de la mairie de...

Friss D'ENREgistrement. - Les frais de timbre et d'enregistrement du présent cahier des charges et des conventions annexées seront supportés par le concessionnaire.

\section{LES INSTALLATIONS HYDRO-ÉLECTRIQUES DE HEIMBACH}

La Rurtalsperrengesellschaft (Société du barrage de la Rur), formée sur l'initiative de la municipalité d'Aix-laChapelle, avec le concours des administrations des villes et des districts de Düren, Schleiden, Heinsberg, Juliers et Montjoie, a pour but la création de divers barrages dans le bassin de la Rur (ou Roer), affluent de la rive droite de la Meuse qu'il ne faut pas confondre avec l'affluent de la rive gauche du Rhin qui porte le même nom. De ces projets, un seul a pris corps jusqu'à présent ; c'est celui du barrage de l'Urft, rivière qui, après avoir serpenté dans le massif montagneux de l'Eifel (Prusse Rhénane), vient se jeter dans la Rur non loin de Ruhrberg,et débite annuellement environ 160 millions de mètres cubes d'eau. Son bassin hydrologique s'étend sur une superficie d'environ 375 kilomètres carrés, dans une région où la hauteur pluviométrique varie entre 800 et 1000 millimètres, ce qui permet d'estimer à 350 millions de mètres cubes le volume annuel des eaux pluviales qui s'y précipitent.

Le barrage a été construit un peu à l'est et en amont du confluent de l'Urft avec la Rur, dans une vallée de trois cents mètres de largeur. C'est un des plus grands parmi les travaux similaires exécutés en Europe. Le double but poursuivi est, en créant un énorme réservoir, de régulariser le régime des eaux d'aval, et de relever le niveau des eaux. d'amont de manière à utiliser l'énergie potentielle résultant de la différence des niveaux, pour alimenter une puissante usine hydro-électrique, située à Heimbach, et destinée à distribuer le courant électrique dans toute la région comprise entre Düren, Aix la-Chapelle et Schleiden.

Le barrage proprement dit est constitué par un mur courbe de $200 \mathrm{~m}$. de rayon, tournant sa convexité vers le lac et mesurant $58 \mathrm{~m}$. de hauteur maxima au-dessus des fondations, $226 \mathrm{~m}$. de longueur et $50 \mathrm{~m}$. 50 d'épaisseur à Ja base. Le couronnement, muni de parapets, supporte une chaussée qui a $5^{\mathrm{m}} 50$ de large et est dallée de basalte. Ce dallage est posé sur une couche de béton de manière à protéger efficacement le mur contre l'infiltration des eaux de pluie, De plus, par suite de linclinaison de la surface de la route, les eaux n'y séjournent pas mais s'écoulent aussitôt vers le lac. Enfin, sur une hauteur de $34 \mathrm{~m}$. à partiı de la base, s'élève contre le parement amont un massif de remblais en terre, incliné à $2 / 3$ et ayant $53 \mathrm{~m}$. de largeur à la base $\left(^{*}\right)$.

Encaissé entre les collines boisées, connues sous le nom de Kermetergebirge. dont l'altitude atteint $520 \mathrm{~m}$., le lac créé par le barrage s'étend jusqu'à Gemünd,c'est-à-dıre sur une distance d'environ $10 \mathrm{~km}$. Sa profondeur maxima, au moment des plus hautes eaux, atteint $52^{\mathrm{m}} 50$, et sá capacité est de 45.500.000 mètres cubes. Circonstance heureuse pour un si grand réservoir, le fond est composé uniquement de roches et de schistes argileux compacts, il est donc parfaitement imperméable.

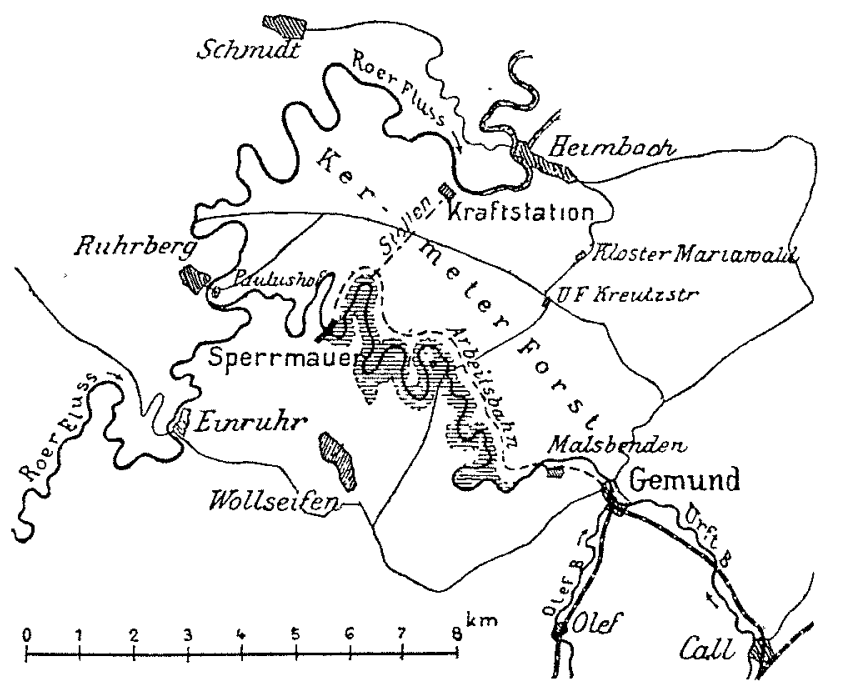

Fı́. 1.- Plan général du barrage (Sperrmauer), du tunnel (Stollen), et de l'usine génératrice (Krafstation).

Gemünd se trouve à une altitude de $338 \mathrm{~m}$. au-dessus du zéro normal officiel allemand. Ce zéro normal, situé à $37 \mathrm{~m}$. au-dessous du point de repère des altitudes normales de l'observatoire de Berlin, correspond sensiblement au niveau moyen de la mer du Nord à Amsterdam. La crête du mur est à la cote 324 , mais on a creusé dans le roc, all flanc de la colline, à l'extrémité nord du mur, les gradins d'un déversoir dont le seuil se trouve à la cote 322,5 , de sorte que l'eau commence à s'écouler dès qu'elle atteint ce niveau, formant ainsi une cascade de $52 \mathrm{~m}$. de hauteur.

Au-dessus du déversoir, le mur est prolongé par un pont de dix travées, de $9 \mathrm{~m} 65$ chacune, ce qui porte la longueur totale du barrage à $326 \mathrm{~m}$. Deux travées sont pourvues de vannes qui permettent d'évacuer les eaux à partir du niveau de $320^{\mathrm{m}} 50$ quand il y a lieu de craindre une crue. Ce dispositif est complété par trois évacuateurs fermés pal des vannes que l'on mancuvre du haut de puits dont les sommets, émergeant des eaux du lac, se trouvent à la hauteur de la crête du mur. Deux de ces évacuateurs sont constitués par des tubes en fer galvanisé, de 0 m60 de diamètre, qui sont placés dans des galeries voûtées traversant la base du mur et le terre-plein établi derrière celui-ci. Le troisième est formé de deux tubes d'acier, de 0m70, qui sont noyés dans le béton, dont on a rempli la galerie qui a servi à détourner les eaux de l'Urft pendant la construction de l'ouvrage.

Le barrage fut achevé en 1904, et l'eau admise dans le réservoir au mois de décembre de la même année. Le 12 janvier 1905 , il contenait 24.500000 mètres cubes, l'eall s'élevant à $40 \mathrm{~m} 50$ de hauteur; le $1^{\mathrm{er}}$ mars, il était rempli, et l'eau alteignait sa hauteur maxima de $52^{\mathrm{m}} 50$.

Du point du lac qui est le plus voisin de Heimbach, l'eall est conduite vers l'usine par un tunnel de $2800 \mathrm{~m}$. de longueur et $6 \mathrm{~m}^{2}$. 14 de section, percé en ligne droite dans la montagne (voir fig. 1). Pour éviter l'entrainement des boues, ce tunnel prend naissance à la cote 284 , c'est-à-dire à une hauteur suffisante au-dessus du fond. Sa pente, qui est

\footnotetext{
(') Pour le profil de ce barrage, voir La Houille Blanche de juin 1900.
} 
particulièrement faible, n'est guère que de 0,36 pour 1000 ou, plus exactement, de $1: 2750$. La paroi en béton, qui forme une voûte en plein cintre à la partie supérieure, a généralement une épaisseur de $28 \mathrm{~cm}$. ; néanmoins, à cause de la nature des terrains traversés, on a porté cetle épaisseur à $50 \mathrm{~cm}$. et même à $77 \mathrm{~cm}$. à certains endroits. La galerie débouche finalement dans un puits, creusé dans le roc depuis la cote 334 jusqu'à celle de 277 , et desliné à amortir les coups de bélier conjointement avec les disposilifs de sécurité installés à cet effet dans l'usine.

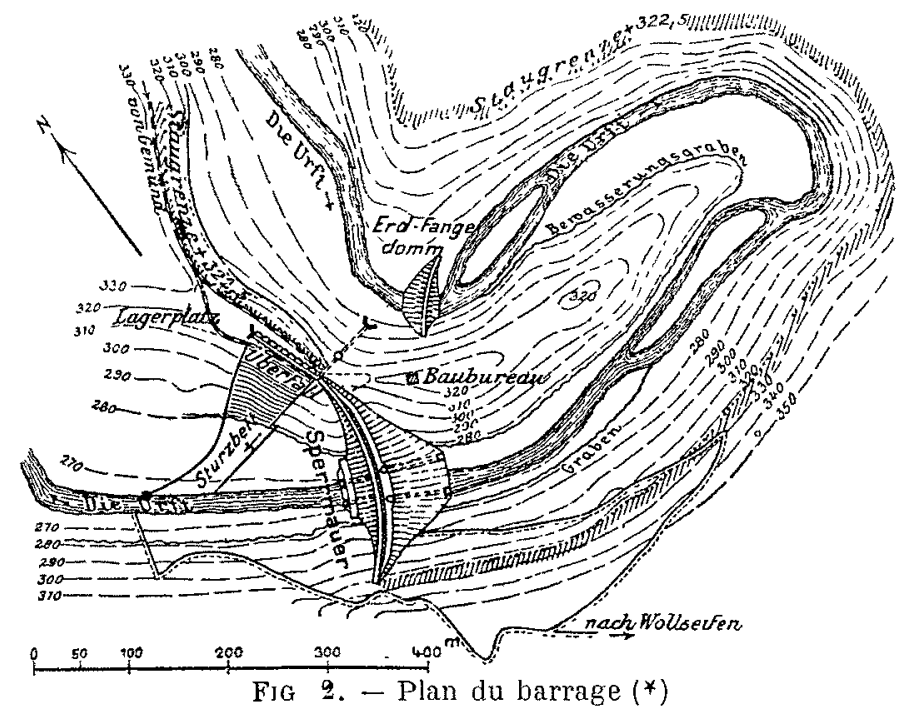

Dans le même puits, s'amorce d'autre part la conduite rorcée, formée de deux tubes d'acier, de 1 m50 de diamètre et de $20 \mathrm{~mm}$. dépaisseur, fermés chacun par un clapet suivi, à peu de distance. d'une vanne oblique. Ces obturateurs sont man'euvrés indépendamment du haut du puits. Les tubes, couchés horizontaleinent côte à côte dans le tunnel, sur une distance de $70 \mathrm{~m}$., sont noyés dans le béton. Les $150 \mathrm{~m}$. restants sont posés dans une tranchée dont le fond fail avec l'horizontale un angle d'environ $30^{\circ}$.

Au pied du coteau, les tubes divergent, pour se diriger ensuite parallèlement vers l'usine avec un écartement de $21 \mathrm{~m}$. d'axe en axe, el y pénétrer le long des murs extérieurs. Immédiatement avant d'entrer dans la salle des machines, ils s'èlargissent momentanément pour recevoir des papillons de forme lenticulaire, en acier coulé, ayant $1^{\mathrm{m}} 80$ de diarnètre, et mobiles autour d'un axe vertical. Ces vannes sont actionnées par des pistons différentiels dont les cylindres sont supportés par les tubes mêmes. L'eau sous pression nécessaire est prise habituellement sur l'une ou l'autre des conduites torcées, mais, en cas de besoin, elle peut être obtenue an moyen d'une pompe à bras. Sous la saile des machines, les conduites reposent sur un lit en béton; elles se terminent par une calotte arrondie qui s'appaie sur l'extrémité d'une poutre horizontale en fer profilé, par l'intermédiaire de laquelle la poussée est transmise à un énorme bloc de béton. A chaque conduite d'amenée correspond un canal de fuite dont les eaux se deversont directement dans la Rur, au sortir de lusine.

Le plan d'eau dans le canal de fuite étant à la cote 211,6 , on dispose, par conséquent, d'une chute brute d'au moins $70 \mathrm{~m}$. à l'étiage, et de $110 \mathrm{~m}$. au moment des hautes eaux et, comme le débit est d'environ 10 mètres cubes par seconde, la puissance disponible est de 7 à 11.000 chevaux

L'usine couvre une superficie d'environ 1000 mètres carrés. Par la porte de la façade principale, située du côté de la rivière, on pénètre de plein-pied dans une vaste salle

(") Cette figure et la précédente sont extrastes de la brochure Dic Urft-Talsperre bei Gemund. LENz, éditeur à Gemund. de $30 \mathrm{~m}$. de long, sur $23 \mathrm{~m}$. de large, où les groupes électrogènes sont rangés sur deux files (fig. 3 et 1 ). Pour faciliter la surveillance et le service des machines, on a installé les alternateurs au centre et les turbines exlérieurement. Colto disposition laisse, le long des murs, de chaquo coté, ainsi qu'au centre, de larges passages pour se rendre au tahloau de distribution qui est installé au fond de la salle, sur uno tribune à laquelle on accède par un double escalier.

Un pont roulant de 8 tonnes, commandé par tirago au moyen d'une chaìne, circule au-dessus du hall.

Les appareils du tableau qui sont à portéc de la main sont tous à basse tension, toutes los comnexions étant établies derrière le tableau et sous la tribuno, dans la chambre ou aboutissent les càbles venant des machnnes et où l'on trouve beaucoup de place disponible pour loger les résistances, etc. Sur les côtés de la dite chambro, au niveau de la salle des machines, il y a un bureau ol un masasin et, dans une amnexe adossee au batiment principal, un atelicr de réparation. Enfin, la partic postéricure de l'usine couvrant lile superficie d'environ 130 mètros cares, est occupćc au rez-de-chaussée par la salle des transfolmateur's et par une chambre de demontage pour ces derniers.

Les dispositifs de protection, tels que paratoulies et bubines de réactance, sont reportés a l'étage supéricur"; enfin, l'usine est flanquée de deux tours qui sont ulilisées pour le départ des lignes. Les transformateurs sont disposćs à la suite l'un de l'autre sur une rangréc; ils sont connectés en étoile, et élèvent la tension, mesurće entre deux conducteurs, de 5.000 à 35000 volts.

La société Fellen el Guilleaume-tahmeyerworko A.-G., de Francfort, ayant recu la commande de la loumiture complète des machines, les ateliers Escher Wyss et Cie, do Zurich, se chargèrent, comme sous-traitants, d'en exúculer la partie hydraulique ; l'appareillage des lignes ot les transformateurs sont dûs à la maison siemens-Schuliert de Berlin.

Turbines. - Les ateliers Escher Wyss et Cie ont adopté des turbines Francis à réaction (fig. 5), calculées pour four: nir, en tournant à 500 tours par minute, 2.000 chevaux quand la chute est de 110 unctres of 1.550 chovaux yluand

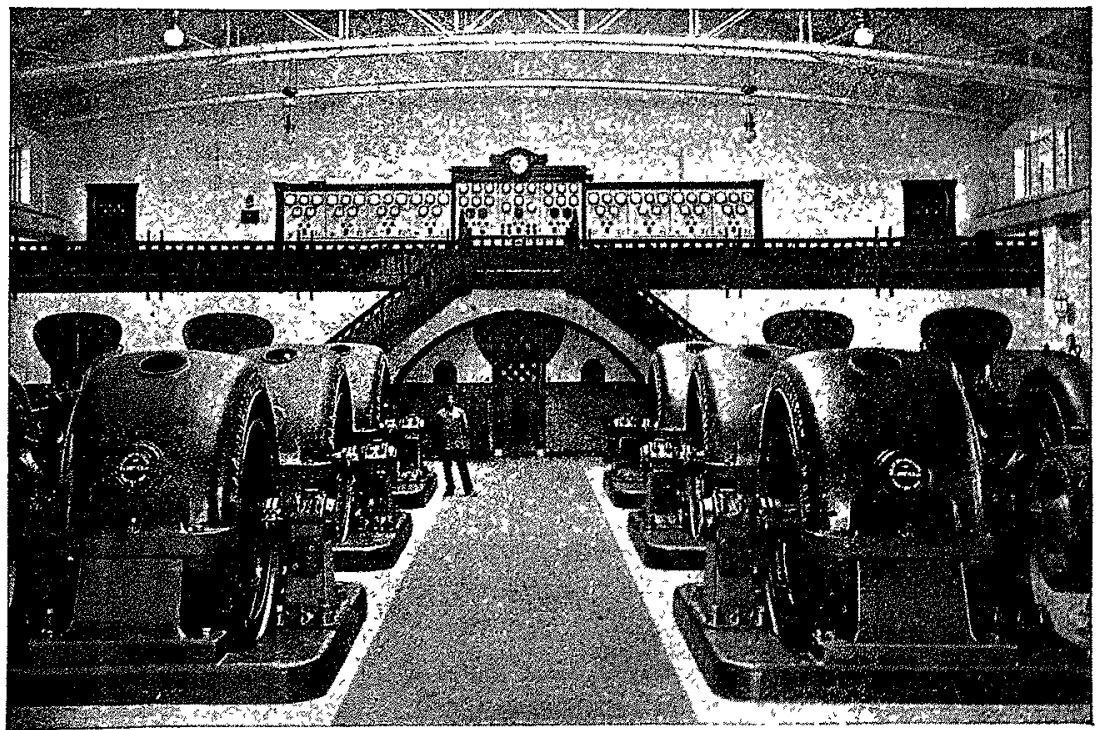

Fia. 3. - Vue intérieure de la centrale le Hermbach.

elle tombe a 70 inotres. Ces tulbines, qui pessont environ 15 tonnes avec leurs accossoires, sont ax axe horizontal et comportent deux roues motrices de $950 \mathrm{~mm}$. de diametre extérieur, en bronze inanganésé de premicre qualite, clavetées sur un arbre en acier qui est renforcé dans sa parlic médiane. Les couronnes des aubes mobiles, ot les aubes directrices qui les entourent, sont enfermés dans une enveloppe en spirale, en fonte, munie de fortes entrotoises. Les aubes directrices, système Finck, sont en acier ; elles 
peuvent pivoter autour de petits axes, et permettent, par conséquent, de modifier l'admission de l'eau à volonté automatiquement ou à la main. Elles sont mues au moyen de deux anneaux en fer lorgé raidis par des traverses radiales, et sont reliées au servo-moteur par un système de bielles et de leviers agissant aux extrémités d'un même diamètre.

Chaque turbine est raccordée à la conduite principale par un coude conique, de $800 \mathrm{~mm}$. de diarnètre, dont la tubulure horizontale contient la vanne d'admission à commande sion. Seul, le cylindre hydraulique qui commande le mouvement de la vanne d'admission est situé au-dessus du sol. La disposition adoptée facilite beaucoup la surveil. ance ainsi que laccès aux différentes parties de la machine.

La régulation de la vitesse et de la puissance motrice est sous la dépendance d'un régulateur à force centrifuge, très sensible, qui reçoit son mouvement de l'arbre de la turbine par poulies et courroie. Quand le manchon du régulateur se lève ou s'abaisse, il déplace la soupape de réglage, c'est.

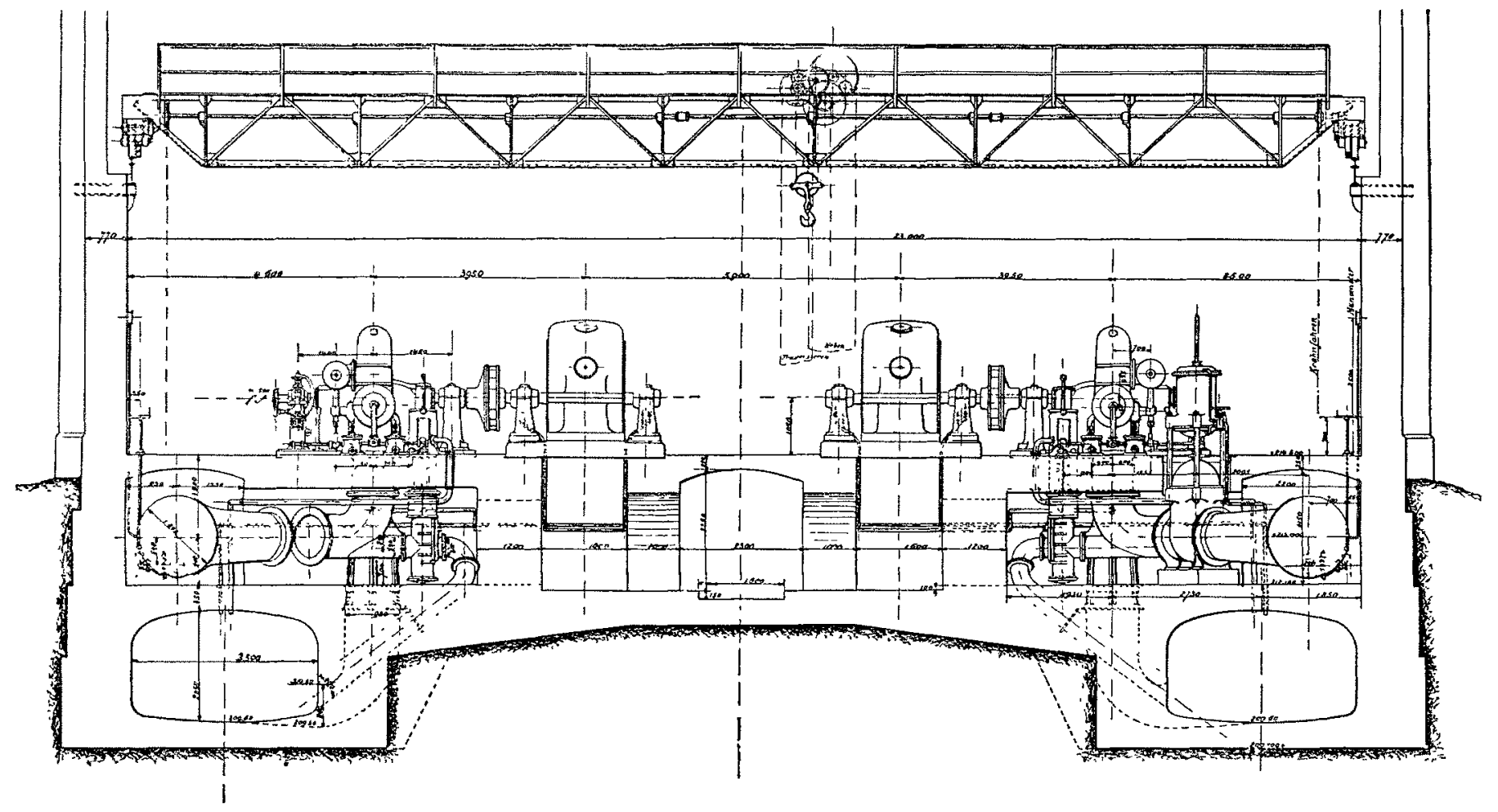

FIG. 4. - Coupe transversale de la'salle des machines.

hydraulique (fig. 4). L'eau pénètre dans les turbines par le bas, parcourt radialement les aubages directcurs et ceux des couronnes molbiles, et en sort axialement de cha que côté, pour se déverser dans le canal de fuite par deux coudes boulonnés sur l'enveloppe spiraloïde et sur la plaque d'assise. Ces coudes contribuent, avec les pieds des deux paliers graisseurs à bagues qui soutiennent larbre,

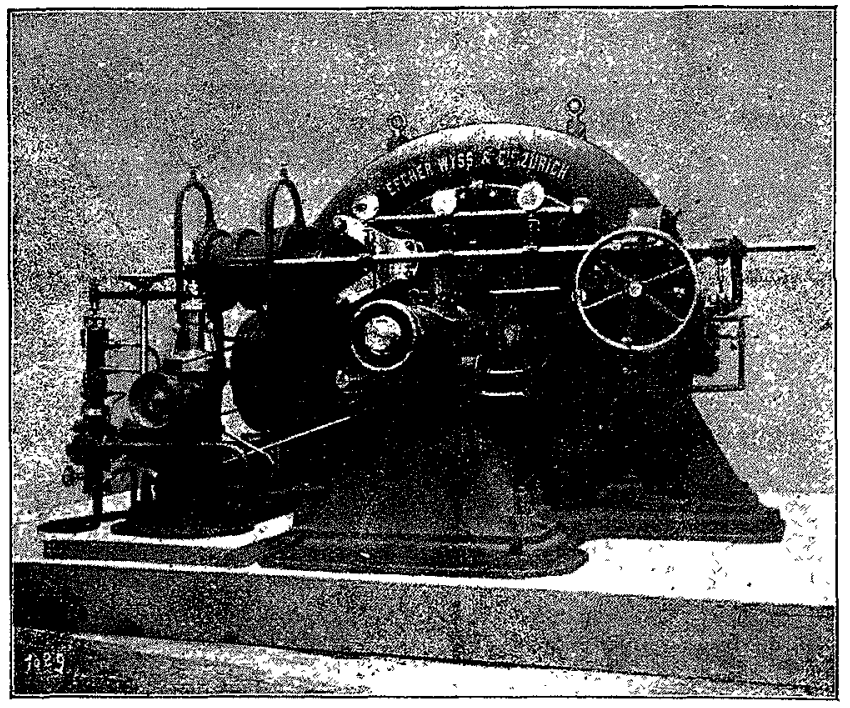

Fig, 5 - Turbıne Francıs à réaction avec son régulateur à servo-moteur.

à augmenter la surface d'appui et à donner à la turbine une solide assiette sur la fondation.

Toute la tuyauterie se trouve sous le sol de la salle des machincs, de même que le dispositif régulateur de pres- à-dire la soupape d'admission du cylindre du servo-moteur, par l'intermédiaire d'un levier du premiér genre, ayant son point d'appui articulé à l'extrémité supérieure d'une tige dont le rôle sera expliqué plus loin. La soupape livre passage à l'eau sous pression qui est envoyée,suivant le cas, sur l'une ou l'autre des faces du piston du servo. moteur dont le cylindre est boulonné sur l'enveloppe spiraloïde (fig. 5). La tige du piston agit alors sur le méca. nisme de réglage des aubes directrices pour modifier l'admission selon la position de la soupape régulatrice. Une manivelle, habituellement débrayée, permet aussi de modifier l'admission à la main par l'action du même mécanisme.

Ce réglage automatique est sulfisant pour les cas ordinailes, c'est-àdire pour parer aux variations do vitesse Hovenant de diminution ou d'aurmentation momentanées de la charge. Mais, quand il s'agit de coupler les alternateur's en parallèle, il faut un réglage plus précis. Dans ce but, la tige dont il a été parlé plus haut peut être levée ou abaissée un peu, de manière à déplacer la soupape régulatrice suivant les besoins. Le mouvement de la tige s'obtient à la main, ou par l'action d'un petit moteur électrique commandé du tableau même de distribution.

Pour parer aux coups de bélier qui ne manqueraient pas de se produire par suite de variations plus ou moins brusques dans l'alimentation, il y a, pour chaque turbine, un l'écrulateur de pression dont les organes sont logés dans une fosse sous la salle des machines. Ce dispositif, rigidement relié au mécanisme de régulation de la vitesse, fait communiquer la conduite d'amenée avec le canal de fuite. et permet, par le jeu d'une vanne, de détourner en totalité ou en partie l'cau arrivant à la turbine. Gràce à lui, on peut fermor complètement l'admission d'une turbine en une 
seconde et demi sans aucun danger. La vanne s'ouvre, en ellet, largement aussitôt, et se referme ensuite lentement d'elle-même. Quoique l'eau soit claire, chaque turbine est accompagnée de deux filtres Escher-Wyss, dits filtres.révolvers a cause de la façon dont la vidange en est faite L'un des filtres sert à l'alimentation du ser'vo-moteur', l'autre à celle du régulateur' de pression.

Matériel électrique - Les alternateurs de la Felten el Guilleaume-Lahmeyerwerke A.-G. sont directement accouplés aux turbines par des accouplements élastiques ZodelVoitb L'arbre, en acier de $180 \mathrm{~mm}$ de diamètro, repose sur deux paliers graisseurs dont l'huile est constamment refroidie par une circulation d'eau L'inducteur mobile est massif et claveté sur l'arbre. Par suite de la grande vitesse de rotation, les douze noyaux pôlaires ont dî recevoir une section elliptique (fig. 6). Les bobines magnétisantes sont faites de fil de cuivre de section carrée, guipé à plusieurs

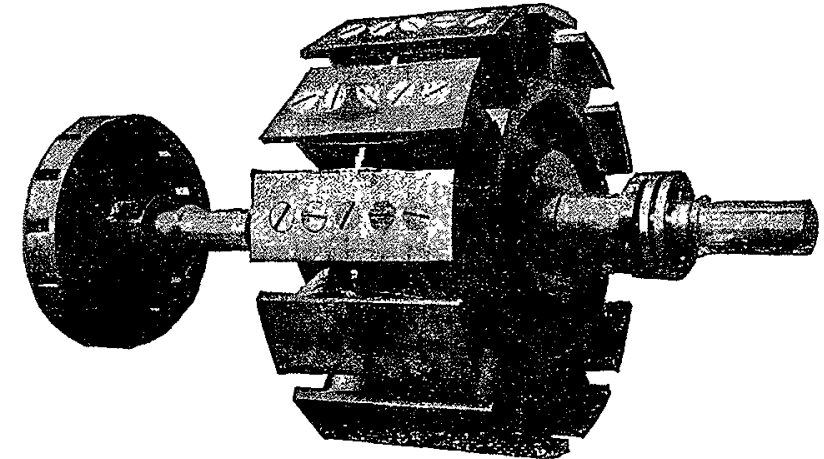

FIG. 6 - Inducteur mobile d'un alternateur de $1600 \mathrm{~K} . \mathrm{V} . \mathrm{A}$.

couches. La longueur du fil et la dépense d'excitation sont bien un peu plus grandes qu'en employant du fil rond, mais, en revanche, les effets de la force centrifuge sont très atténués; la dénudation des fils, en particulier, est rendue très difficile. Les pièces polaires en fer doux sont massives; la construction est ainsi plus simple et plus solide, et l'on évite le ronflement désagl'éable dû aux pôles feuilletés. En outre, l'action amortissante des courants de Foucault, qui prennent naissance dans leur inasse, diminue l'amplitude des mouvements pendulaires lorsque l'alternateur fonctionne en parallèle avec d'autres. Toutefo's, pour éviter un échauffement et une perte donergie excessifs, on a donné une forme convenable aux encoches et aux cornes polaires, de manière à obtenir une répartition homogène du flux dans l'entrefer.

Le noyau en tôles minces de l'armature est logé dans une forte calcasse en fonte, coulée en deux pièces qui sont assemblées ensuite par boulons (fig. 8). Les encoches, demi-fermées, sont garnies de tubes en micanite sans joint, à l'intérieur. desquels le bobinage est tiré à la main. Les têles sont soigneusement faites sur formes, de facon à maintenir partout l'ćcartement maximum enlle les bolsines adjacentes. Au sortir des encoches, les conducteurs sont, en outre, recouverts de ruban isolant, et les croisements sont évités dans la mesure du possible La rupture d'isolement est donc rendue pratiquement impossible entre les divers conducteurs, aussi bien qu'entre les bobinages et le fer. Les bobinages sont protégés contre tout contact extérieur par des couronnes perforées en fonte.

Mentionnons aussi que ces machines reposent sur leur fondation par un système de calago réslable, très commode quand il s'agit de rattraper l'usure des coussinets. L'enveloppe de fonte est percée de nombreuses fenêtres de ventilation qui peuvent être fermées à volunté, ce qui permet d'agir suivant les besoins sur le refroiảissement des différentes parties de la machine.

Les deux excitalrices (fig. 7) sont des dynamos shunt, type E. V. de la Fellen et Guilleaume-Lahmeyerwerke.Elles ont une puissance de $135 \mathrm{kw}$, sous une tension de 225 volts, et tournent à 900 tours par minute. Elles sont munies de pôles compensaleurs, de sorte que la commutallon a licu sans étincellec, quelle que soit la cllarge De mème que les alternateurs, les excitatrices sont actionnes par des turbines Francis à réaction, de la maison lischer-lVyss, mais celles-ci sont de construclion plus simple, en ce sens qu'elles ne comportent qu'une seule couronne d'aubes mobile, de $560 \mathrm{~mm}$. de diamétro extérieur, el qu'clles no sont pas munies de régulateur de pression. Leur puissance est de 208 chevaux. Le raccord de prise d'eau n'a que $300 \mathrm{~mm}$. de diamètre et la vanue d'admission est actionnce ì la main.

Les données principales concernant les alternateurs et les excilatrices sont résumées dans lo tableau suivant:

\begin{tabular}{|c|c|c|}
\hline & ALTERNATEURS & EXCITATRICES \\
\hline Puissance. & $1600 \mathrm{~K} . \mathrm{V} . \mathrm{A}$ & $135 \mathrm{kiv}$ \\
\hline Tension..... & 5400 volts & 225 volts. \\
\hline Vilesse de rotation...... & 500 tours & 900 tours \\
\hline Diamètre inlérieur de l'in- & 69501 & 530 \\
\hline Longueur de l'induit..... & $800 \mathrm{~mm}$ & $220 \mathrm{~mm}$ \\
\hline Alèsane de linduit....... & $1700 \mathrm{~mm}$. & $226 \mathrm{~mm}$ \\
\hline
\end{tabular}

Dianètre extérieur de l'in-

ducteur volant....... $1662 \mathrm{~mm}$.

Diamètre extérieur de la carcasse.

Nombro de pôles...........

Résistance de l'induit....

Poids du rotor, environ..

Poids total de la machine,

y compris les paliers, l'ar'-

bre et le socle, environ.
12

$0,131 \mathrm{olim}$.

$7700 \mathrm{kgs}$

$33000 \mathrm{kgs}$
$1500 \mathrm{~mm}$. 4

0,0066 ohm . $850 \mathrm{kgs}$.

Les essais de réception, fui curent lieu du 4 au 8 septembre 1905 , donnèrent pleine satisfaction. On put notamment constater l'efficacité du régulateur hydrauliruc pour lequel la maison Escluer-Wyss donnait les garanties suivantes : pour des variations brusques de la charge, on plus ou en moins, de $10 ; 25$ et 30 pour 100, la vitesce ne devant pas varier de plus de $2 ; 3$ et 4 pour 100 , et les varialions de la

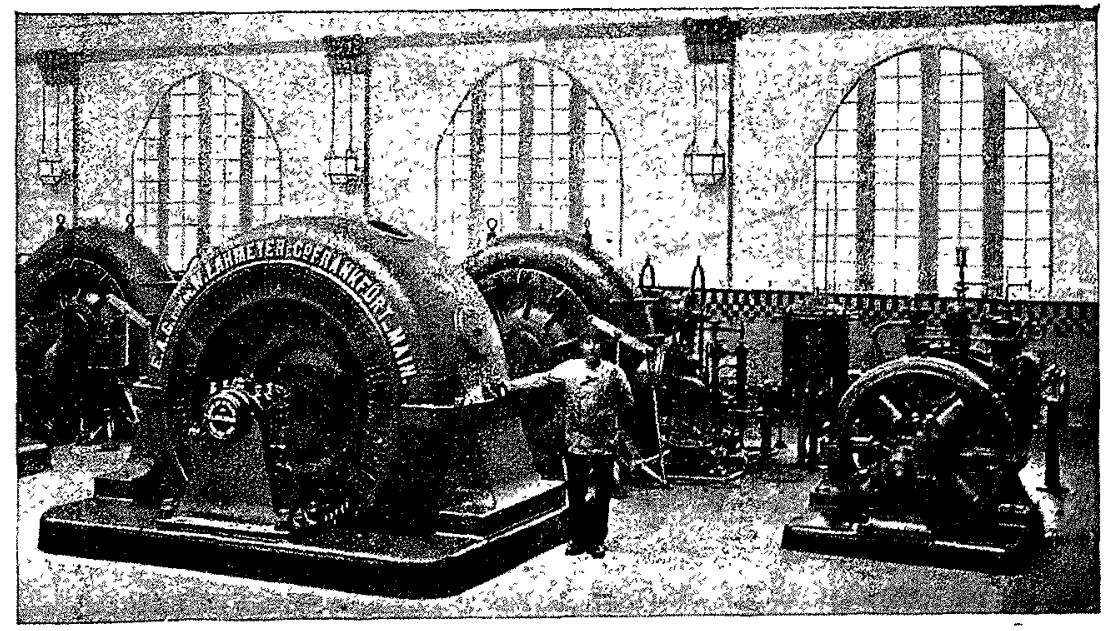

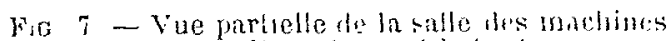
montrant, à gauche un allernatcur, da droste une excilatero.

pression ne devaient pas déjasser 1,5; 3 et 5 pour 100. Les essais au flein accusierent un rendement de $8 \dot{0}, 3$ pour 100 a pleine charere; 77,8 pour 100 a trois-quart do charge et 72 pour 100 a demi-charge.

Depuis cette epoque, l'usine a marché sans interruption et sans accident; nćanmoins, on a decide dy installer, comme réserve, un turbo-alternateur de $2200 \mathrm{kw}$. Au mois de mai de l'an dernicr, la longueur des lignes du réseau à 
35000 volts atteignait 166 kilomètres. Cette tension de 35000 volts est celle des lignes partant de l'usine ; elle est transformée en différents points, et le courant est distribué sous diverses tensions plus basses par des lignes dont la longueur totale atteignait, à la même époque, 238 kilomètres.

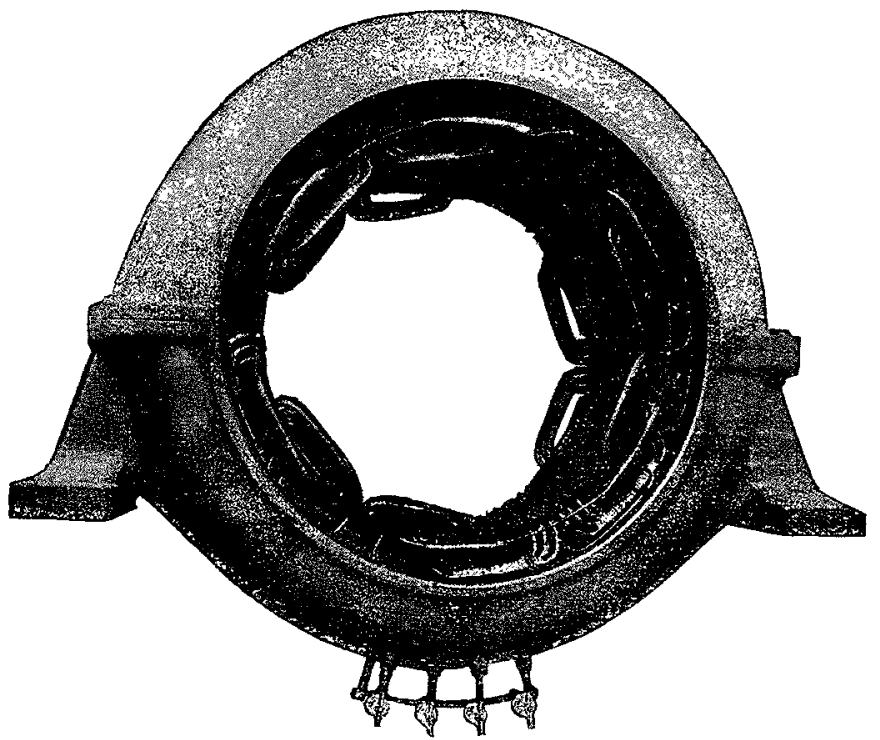

Frg. 8. - Induit fixe d'un alternateur trıphasé de 1600 K.V.A.

Le prix de revient de I'ensemble, lignes comprises, est d'environ 12,5 millions de francs. La création du réservoir a couté 5 millions à elle seule, ce qui met je mètre cube à 0 fr. 11, chiffre le plus bas que nous connaissions, la moyenne des installations hydrauliques d'Allemagne revenant à $0 \mathrm{fr} .375$ le mètre cube.

Les divers centres de répartition paient le kilowattheure entre 4,75 et 5,125 centimes, et le revendent entre 7,5 et 30 centimes, à la tension de 5000 volts. Dans le cas de basse tension, ce prix peut atteindre jusqu'à 44 centimes.

IVan BONIVER. Ingenieur-ëlectricien.

\section{SUR LE ROLE DE LA STRUCTURE GÉOLOGIQUE dans l'évaluation des débits DES TORRENTS ALPESTRES}

Note présentée au Congrès d $\mathrm{l}$ l'Association française pour l'Avancement des Sciences, tenu à Reims, en 1907, au cours de la séance du 3 août, par M.W. KILIAN, professeur à l'Université de Grenoble.

Il convient de mettre en garde contre les erreurs, parfois considérables, que peut provoquer l'appréciation a priori trop sommaire et toute théorique du débit d'un cours d'eau de montagne lorsqu'on ne tient pas compte de la structure du géologique bassin de réception.

S'il existe, en effet, un rapport, qui, dans beaucoup de cas, est constant entre la surface du bassin d'alimentation et le débit moyen d'un torrent alpestre, et si ce rapport peut, lorsqu'on tient compte de la moyenne anmuelle des précipitations annuelles et du coefficient d'évaporalion (I), fournir, dans la plupart des cas, des données précieuses sur le débit utilisable du cours d'eau considéré, cette appréciation ne peut donner des résultats utiles que lorsqu'il s'agit d'un bassin dont les conditions géologiques ne présentent rien d'anormal, et lorsque les limites topographiques du bassin hydrographique coüncident dans leur ensemble avec

(1) Ainsi que de l'état de la surface du sol (boisée ou dénudét).

celles du bassin géologique. Dans d'autres cas, assez nombreux, la structure géologique peut intervenir d'une façon très notable dans l'alimentation du torrent, et peut en modi. fier considérablement le débit, de façon à rendre abso. lument illusoires les évaluations résultant du calcul indiqué plus haut.

Les principaux facteurs géologiques influant dans ce sens sont les suivants :

a) L'inclinaison des couches.

Les eaux d'infiltration provenant, soit des précipitations, soit de la fusion des glaciers, suivent en général, dans leur

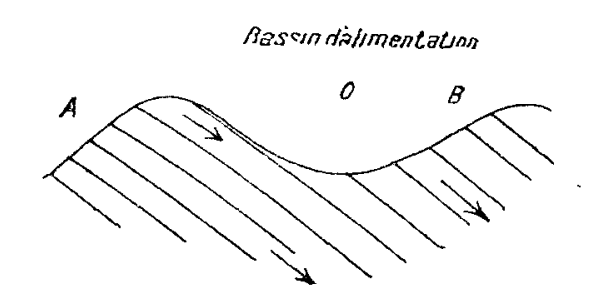

Frg. I. - Le bassin O ne recevra que les infiltrations de la région $\mathrm{AO}$, celle de $\mathrm{OB}$ seront perdues pour lui. trajet souterrain, les joints séparant les couches successives (de perméabilité souvent différente); l'inclinaison des assises, le a pendage» des couches, a donc une importance capitale dans l'alimentation du cours d'eau; la disposition du pendage, par rapport au bassin d'alimenta* tion topographique, peut avoir pour effet, suivant les cas, soit de détourner une partie des infiltrations en dehors de ce bassin ( f $g$. I et 2), soit d'y amener des eaux de dépressions superficielles limitrophes $C$ et $D$, (fig. 4,).

Un simple coup Fig. 2. - Le bassin O recevra un minzmum d'infil. d'œil sur les figures grande paatie entraineées dans des bassins voisins I à 4 permet de se

rendre compte des conditions variées qui peuvent se présenter à cet égard.

Il peut exister, en outre, des dispositions tectoniques plus compliquées, pouvant modifier d'une façon plus importante encore le débit d'un bassin topographique donné.

b) La nature plus ou moins imperméable, fissurée on caverneuse des roches, donnant lieu, suivant les cas, soit à un régime de ruissellement et de filtration souterraine lent et régulier (normal); soit, au contraire, - et c'est le cas surtuut pour les roches calcaires, pour les cargneules et les gýpses - à l'existence d'un peritable réseau hydrogrtphique souterrain : boyaux, cavités, lacs souterrains, parfois reliés par des siphons naturels, torrents souterrains venant parfois émerger au jour par des "résurgences " ou « sources

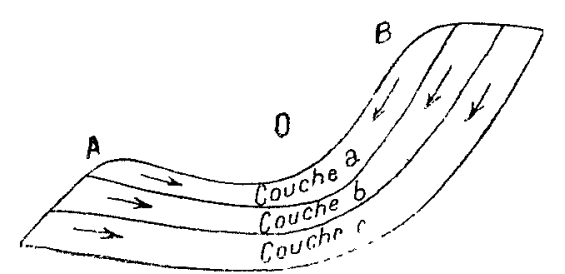

Fla. 3. - Ie bassin $O$ ne sera alimenté que par les irfitrations qu'à reçu, entre $A$ et $B$, la couche superficielle $a$. Les eaux des couches $b$ et $c$ resteront en profondeur. donnent lieu, en outre, en déterminant l'absorption rapide et la descente facile en profondeur des eaux superficielles, qu'elles soustraient ainsi à l'évaporation, à des réserves de liquide inattendues et importantes tout à fait disproportionnées avec le débit que donne un régime de filtration débit. Ces réseaux de fissures des terrains calcaires n'ont parfois aucun rapport avec la stratification des au. tres assises de la région, ni avec les limites topographiques du bassin hydrographi. vauclusiennes ) à fort

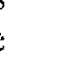

